\title{
A Fresh Breath of Oxygen: Red Blood Cell Exchange Transfusion in Sickle Cell and COVID-19
}

\author{
Viva Nguyen ${ }^{1}$, Paul Alcius ${ }^{1}$, Shachar Peles ${ }^{2}$, and Katherine Hodgin ${ }^{3}$ \\ ${ }^{1}$ University of Miami Miller School of Medicine \\ ${ }^{2}$ Florida Cancer Research \& Research Institute \\ ${ }^{3}$ JFK Medical Center
}

March 24, 2021

\begin{abstract}
We present the first documented case of a 69 year-old female with history of sickle cell anemia with hereditary persistence of fetal hemoglobin that presented due to joint pains and COVID-19 infection. The red blood cell exchange transfusion may play an important factor in preventing intubations and longer hospital stays.
\end{abstract}

Title: A Fresh Breath of Oxygen: Red Blood Cell Exchange Transfusion in Sickle Cell and COVID-19 Authors: Viva Nguyen, $\mathrm{MD}^{1}$, Paul Alcius, $\mathrm{MD}^{2}$, Shachar Peles, $\mathrm{MD}^{3}$, Katherine Hodgin, $\mathrm{MD}^{4}$

Affiliations:

${ }^{1}$ Internal Medicine Resident, University of Miami JFK GME, Atlantis, FL, USA

${ }^{2}$ Internal Medicine Resident, University of Miami JFK GME, Atlantis, FL, USA

${ }^{3}$ Hematologist and Oncologist, Florida Cancer Specialists \& Research Institute, Lake Worth, FL, USA

${ }^{4}$ Critical Care Medicine Intensivist, JFK Medical Center, Atlantis, FL, USA

Corresponding Author: Viva Nguyen, 180 JFK Drive Suite 210, Atlantis, FL, USA, 33462, 561-888-3069, (vhn5@med.miami.edu).

Abstract Word Count: 82Text Word Count: 997

Number of references: 7 Number of tables and figures: 0

Number of supplemental illustrations/tables: 0

Running title: Transfusion in Sickle Cell and COVID-19

Keywords: red blood cell exchange transfusion, sickle cell disease, acute chest syndrome, hereditary persistence of fetal hemoglobin, COVID-19

Key Clinical Message: Red blood cell exchange transfusion may be beneficial to patients with sickle cell disease and COVID-19 early on in the clinical presentation to prevent the need for intubation and intensive care unit admission due to respiratory distress.

Abstract 
We present the first documented case of a 69 year-old female with history of sickle cell anemia with hereditary persistence of fetal hemoglobin that presented due to joint pains and COVID-19 infection. The red blood cell exchange transfusion may play an important factor in preventing intubations and longer hospital stays.

Introduction

SCD is one the most common hemoglobinopathies in the world, with important complications such as vasoocclusive crisis (VOC) and acute chest syndrome (ACS). With the worldwide pandemic of COVID-19, the overlap between clinical presentations of life-threatening pneumonia versus ACS have blurred the lines for treatment. With early treatment, sickle cell patients and others with hemoglobinopathies may benefit from early red blood cell exchange transfusions to prevent deterioration in those with COVID-19 infections.

Case Report

A 69 year-old African American female with past medical history of sickle cell anemia with mild phenotype from hereditary persistence of fetal hemoglobin on folic acid presented to the emergency department (ED) due to joint pain and fevers. She denied any chest pain, shortness of breath, cough, nausea, vomiting, diarrhea. Patient reported that she tested positive for COVID-19 at an outpatient facility. She was admitted for sickle cell crisis, symptomatic anemia with hemoglobin (Hgb) of 6.4, and COVID-19 pneumonia. A unit of packed red blood cells (pRBCs) was ordered and transfused in the ED. Chest x-ray (CXR) upon admission: "Mild perihilar interstitial infiltrates and/or edema, mild peripheral right ground-glass infiltrates. Pneumonia considered." Computed tomography (CT) angiogram of the chest was performed: "Extensive bilateral ground-glass subpleural opacity is seen consistent with COVID pneumonia. No evidence pulmonary emboli." Infectious disease was consulted by the primary team; patient was started on ceftriaxone, azithromycin, and convalescent plasma was ordered on day of admission. Hemoglobin electrophoresis demonstrated: hemoglobin A 92.1\%, hemoglobin A2 2.5\%, hemoglobin C 0.0\%, hemoglobin F 0.0\%, hemoglobin S $5.4 \%$.

Two days after admission, the rapid response team was called due to shortness of breath. Upon initial evaluation, patient was in respiratory distress with use of accessory muscles. Pulse oximeter revealed an oxygen saturation of $87 \%$ on nonrebreather. At that time, the decision was made to transfer the patient to the intensive care unit (ICU) for planned red blood cell exchange transfusion (RBCX). Upon arrival to the ICU, patient's respiratory status was worsening. As a result, patient was intubated and sedated, placed in proning position for 16 hours. Ten units of hemoglobin S negative, leukocyte reduced RBCs were ordered and transfused throughout the night. As a result of the RBC transfusion exchange, another unit of convalescent plasma was transfused the following day. Ultimately, patient was extubated two days after intubation and was transferred out of the ICU the following day after extubation.

\section{Discussion}

Patients with SCD often present to the hospital because of acute pain or symptomatic anemia. Hemoglobin is normally soluble in the erythrocyte and does not polymerize; hemoglobin $\mathrm{S}(\mathrm{HbS})$, resulting from a point mutation in the beta globulin gene, becomes poorly soluble when deoxygenated. ${ }^{1}$ This pathological polymerization of HbS, which forms the classic crescent or sickle shape of erythrocytes, is one of the main causes of VOC. ${ }^{1}$ The degree of polymerization is a determinant of the severity of SCD, which is affected by other hemoglobin mutations co-occurring with $\mathrm{HbS}$ and concentration of fetal hemoglobin.

An important factor in the clinical manifestation and prognosis of our 69 year-old patient is the presence of hereditary persistence of fetal hemoglobin (HPFH). Uniquely, our patient was homozygous for HPFH, which likely contributed the longevity of her life-span. Patients with SCD are immunocompromised, thus avoiding COVID-19 is crucial. Data is limited for the patients with SCD and COVID-19 infection.

Like any other respiratory viruses, COVID-19 may because pneumonia, leading to potential ACS or respiratory failure in those with SCD. ${ }^{2}$ Because symptoms overlap between COVID-19 pneumonia and ACS, any potential deterioration in respiratory status needs prompt intervention. There have been cases reported of COVID-19 causing ACS. ${ }^{3}$ It is hypothesized that a possible mechanism of severe acute respiratory syndrome 
coronavirus 2 (SARS-CoV-2) is that the virus affects the normal, functioning structure of hemoglobin, causing the hypoxemia. ${ }^{4}$ Thus, by directly addressing the lack of oxygen via hemoglobin directly with RBCX, we may prevent rapid declines in oxygenation.

In our patient's situation, her respiratory status deteriorated rapidly to a point where intubation was deemed necessary to protect her airway and to allow her body to rest while she received the RBCX. The goal of an exchange transfusion to significantly lower $\mathrm{HbS}$ levels to 30 percent or less and correct any potential anemia. By lowering the $\mathrm{HbS}$ level, in particular those with acute organ deterioration, we limit sickling complications.

For our patient, she got intubated and then received the RBCX. The patient recovered rather quickly after intubation, just requiring 48 hours on the ventilator, which is uncommon for those with COVID-19 pneumonia and its complications. The RBCX improved her oxygenation status and ultimately led to a quicker recovery. In other case reports, there are have been suggestions of conducting RBCX to patients with SCD and COVID-19 after admission and before rapid respiratory deterioration to prevent intubation and ICU admission. $., 5,6,7$ Our patient had to intubated, then received RBCX, and then received another dose of convalescent plasma due to the RBCX. Perhaps if we were able offer the patient RBCX prior to respiratory distress, we could have prevented the intubation procedure, ICU admission, and the extra dose of convalescent plasma, which are certainly costly in the hospital setting.

Limitations for our proposed "prophylactic" RBCX prior to respiratory deterioration is the limited reported cases, in particular those patients with SCD and COVID-19. Likewise, blood bank shortages may prevent the possibility of RBCX at a given facility. The transfer of potential patients to facilities that could conduct an RBCX could be timing consuming and costly as well.

\section{Conclusion}

This case report demonstrates that a well-known procedure of RBCX may be beneficial to patients with SCD and COVID-19 early on in the clinical presentation to prevent the need for intubation and ICU admission due to respiratory distress. Clinical improvement with RBCX appears to be beneficial, as well as the benefit of potentially decreasing financial costs and limiting sparce resources, such as ICU beds and staffing during a worldwide pandemic. Further studies will need to be conducted to validate benefits of early RBCX in SCD and COVID-19 patients.

\section{Conflicts of Interest}

The authors declare that they have no conflicts of interest.

\section{Patient Consent}

Written informed consent for patient information and images was provided by the patient.

\section{References}

1. Bunn, H. F. (1997). Pathogenesis and Treatment of Sickle Cell Disease. New England Journal of Medicine , 337 (11), 762-769. https://doi.org/10.1056/nejm199709113371107

2. Sivalingam, T., Inusa, B., Doyle, P., \& Oteng-Ntim, E. (2020). COVID-19 and the pulmonary complications of sickle cell disease.EJHaem , 1 (2), 545-547. https://doi.org/10.1002/jha2.105

3. Beerkens, F., John, M., Puliafito, B., Corbett, V., Edwards, C., \& Tremblay, D. (2020). COVID19 pneumonia as a cause of acute chest syndrome in an adult sickle cell patient. American Journal of Hematology , 95 (7), E154-E156. https://doi.org/10.1002/ajh.25809

4. Hacking, S. M. (2020). Red blood cell exchange for SARS-CoV-2: A Gemini of therapeutic opportunities. Medical Hypotheses ,144 , 110227. https://doi.org/10.1016/j.mehy.2020.110227

5. Hussain, F. A., Njoku, F. U., Saraf, S. L., Molokie, R. E., Gordeuk, V. R., \& Han, J. (2020). COVID-19 infection in patients with sickle cell disease. British Journal of Haematology , 189 (5), 851852. https://doi.org/10.1111/bjh.16734 
6. Allison, D., Campbell-Lee, S., Crane, J., Vidanovic, V., Webb, S., Fraidenburg, D., \& Hussain, F. (2020). Red blood cell exchange to avoid intubating a COVID-19 positive patient with sickle cell disease? Journal of Clinical Apheresis , 35 (4), 378-381. https://doi.org/10.1002/jca.21809

7. Okar, L., Aldeeb, M., \& Yassin, M. A. (2021). The role of red blood cell exchange in sickle cell disease in patient with COVID-19 infection and pulmonary infiltrates. Clinical Case Reports , 9 (1), 337-344. https://doi.org/10.1002/ccr3.3526 